\title{
Recent results of inclusive and differential top quark pair production cross section measurements at CMS
}

\section{Burns* on behalf of the CMS Collaboration}

Department of Physics, University of Bristol

IIHE, Vrije Universiteit Brussel

E-mail: Douglas.Burnsabristol.ac.uk

\begin{abstract}
Latest results on inclusive top quark pair production cross sections are presented using protonproton collision data collected by CMS at different centre-of-mass energies, including $5 \mathrm{TeV}$. Final states with at least one charged lepton and one b-jet are explored to measure the inclusive production cross sections. The first top quark pair production cross sections measurements in proton-lead collisions are discussed. The first differential top quark pair production cross sections using data collected by CMS in 2016 are discussed with respect to kinematic variables in final states with at least one lepton.
\end{abstract}

ICHEP 2018, International Conference on High-Energy Physics

4th - 8th July 2018

Seoul, Korea

${ }^{*}$ Speaker. 


\section{Introduction}

Measurements of inclusive and differential top quark pair (tit) production cross sections provide important tests of the standard model (SM). Such measurements also allow the determination of SM parameters and constrain the parton distribution functions (PDFs) of the proton, in particular, the gluon distribution function. At the collision energies of the LHC this means it is primarily used to constrain the gluon distribution function of the proton. On top of being sensitive to the initial state of the proton it is also sensitive to soft QCD radiation emitted in both the initial and final state. This means that it can provide information for the basis of better parton shower modelling. A further advantage of the t⿱t production cross section is that it is sensitive to new physics beyond the SM (BSM). This may occur either though the direct production of top quark pairs from a BSM particle, through the production of BSM particles in its decay products or through its couplings, for example in an effective field theory. The $t \bar{t}$ final state is also present as a background to many BSM and rare SM searches.

The tit production cross section can be measured inclusively or differentially (as a function of a kinematic variable) with respect to many different final states. These include the three physical categories where the $\mathrm{W}$ boson decays to a quark-antiquark pair or lepton-neutrino pair resulting in the 'dilepton', 'single lepton' and 'all-hadronic' final states. They can be measured with respect to the constituent partons hypothesised in the collision, known as 'parton level', with respect to stable particles (mean lifetime $>30 \mathrm{ps}$ ) produced after the shower modelling, known as 'particle level' or with respect to the raw detector output, known as 'reconstruction level'. They can be measured in a 'visible phase space' that is similar to that accessible by the CMS experiment [1] or extrapolated to the 'full phase space'.

\section{Inclusive cross sections}

The first inclusive $t \bar{t}$ cross section measurement presented here represents the first measurement using 5.02 $\mathrm{TeV}$ proton-proton collision data from the CMS experiment [2]. It is performed using the combined single lepton and dilepton channels, where the dilepton channel contains at least one muon. In the former channel, $\sigma_{\mathrm{pp} \rightarrow \mathrm{tt}}$ is extracted using a simultaneous fit of the angular distribution $\left(\Delta R_{j j^{\prime}}\right)$ between all pairs of jets $\left(j, j^{\prime}\right)$ for events containing $0 \mathrm{~b}$ quarks, $1 \mathrm{~b}$ quark, and $>2 \mathrm{~b}$ quarks. The value of $\sigma_{\mathrm{pp} \rightarrow \mathrm{tt}}$ in the full phase space is determined to be $68.9 \pm 6.5$ (stat.) \pm 6.1 (syst.) \pm 1.6 (lumi.) pb. For the latter channel, $\sigma_{\mathrm{pp} \rightarrow \mathrm{tt}}$ is extracted using a cut-and-count method. Events are counted in the same visible phase space as for the single lepton channel and the cross sections extrapolated to the full phase space. The value of $\sigma_{\mathrm{pp} \rightarrow \mathrm{tt}}$ in the full phase space is determined to be $77 \pm 19$ (stat.) \pm 4 (syst.) \pm 2 (lumi.) pb for events containing $e^{ \pm} \mu^{\mp}$ and 59 \pm 29 (stat.) \pm 11 (syst.) \pm 1 (lumi.) pb for events containing $\mu^{ \pm} \mu^{\mp}$. All cross sections measured are combined using the BLUE method resulting in a value for $\sigma_{\mathrm{pp} \rightarrow \mathrm{t}}$ of $69.5 \pm 6.1$ (stat.) \pm 5.6 (syst.) \pm 1.6 (lumi.) pb. All measurements are shown in the left panel of Fig. 1 and are consistent with the SM.

The second inclusive $t \bar{t}$ cross section analysis presented here represents the first observation of top quarks using 8.16 TeV proton-lead collision data from the CMS experiment [3]. The measurement is performed in the single lepton decay channel and $\sigma_{\mathrm{pPb} \rightarrow \mathrm{tt}}$ is extracted from a combined 
likelihood fit of the di-jet invariant mass $\left(M_{j j^{\prime}}\right)$ for events containing $0 \mathrm{~b}$ quarks, $1 \mathrm{~b}$ quark, and $>2$ $\mathrm{b}$ quarks. A total of $710 \mathrm{t} \overline{\mathrm{t}}$ signal events are measured, with the largest uncertainty originating from the $b$ quark tagging efficiency. The background only hypothesis is rejected with a significance over 5 standard deviations. The value of $\sigma_{\mathrm{pPb} \rightarrow \mathrm{tt}}$ in a final state containing one muon is measured to be $44 \pm 3$ (stat.) \pm 8 (syst.) nb and containing one electron to be $56 \pm 4$ (stat.) \pm 13 (syst.) nb, resulting in a combined $\sigma_{\mathrm{pPb} \rightarrow \bar{t}}$ of $45 \pm 8$ (tot.) nb. The measured cross sections are shown in the right panel of Fig. 1 and are compatible with theoretical predictions.
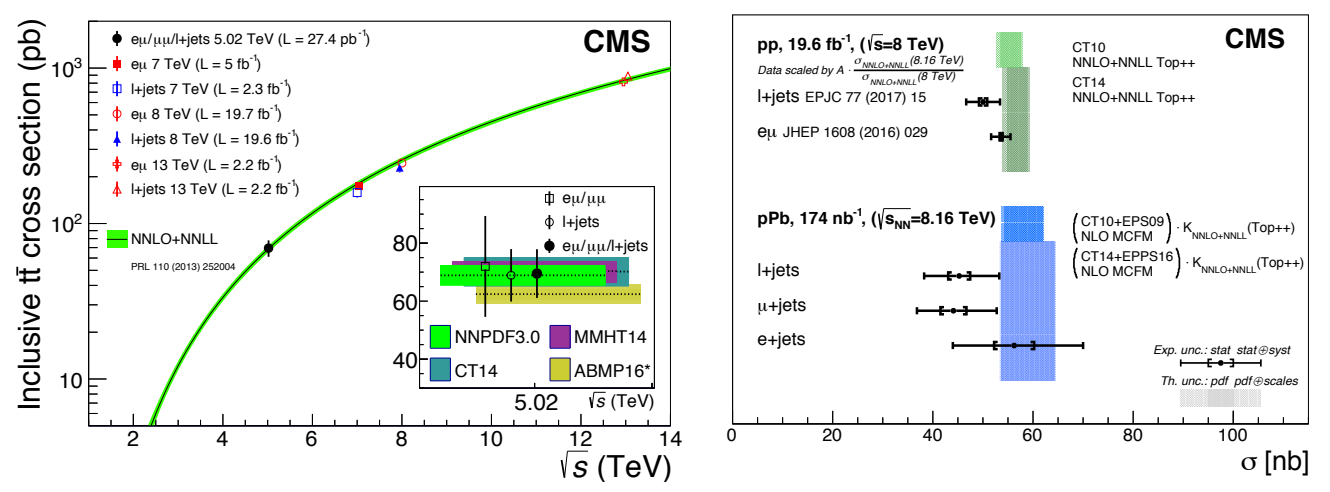

Figure 1: The left panel shows the inclusive cross section measurement for all accessed $\sqrt{s}$, highlighting measurement at $5.02 \mathrm{TeV}$. The right panel shows the additional constraints placed on the gluon PDF by the inclusion of $5.02 \mathrm{TeV}$ inclusive cross section measurements. Figures taken from [2].

\section{Differential cross sections}

The first cross section analysis presented here forms the first differential $\sigma_{\mathrm{pp} \rightarrow \mathrm{tt}}$ paper produced by CMS using the 2016 data set [4]. Normalised and absolute cross sections are measured with respect to kinematic event variables (do not require $t \bar{t}$ system to be reconstructed) in the single lepton decay channel and is presented to particle level in a visible phase space. Comparisons are performed with several state-of-the-art leading order (LO) and next-to-leading order (NLO) generators using $\chi^{2}$ goodness-of-fit tests. The left panel of Fig. 2 shows the absolute cross section with respect to the hadronic activity $H_{\mathrm{T}}$. It displays a trend, consistent for variables correlated to the top quark $p_{\mathrm{T}}$, of being softer in data than in simulation. The NLO generators are concluded to be generally consistent with the data, although no one generator describes the data perfectly and the LO generator does not describe the data.

The second cross section analysis presented here also uses the full 2016 CMS dataset [5]. It operates in the same channel as the previous analysis and includes normalised and differential cross section measurements with respect to variables relating to the kinematic properties of the reconstructed event and to additional jets. They are presented at both particle level in a visible phase space and parton level in a full phase space. The centre panel of Fig. 2 shows the absolute $\sigma_{\mathrm{pp} \rightarrow \mathrm{t}}$ with respect to additional jet multiplicity, which agrees very well with the NLO generators. Double differential measurements are also performed, for example the complete kinematic properties of the top quark $\left(p_{\mathrm{T}}\left(t_{h}\right),|\eta|\left(t_{h}\right)\right)$. The analysis also finds that no generator describes the data 
perfectly and the observed trend in variables correlated to the top quark $p_{\mathrm{T}}$ is consistent with other measurements.

The final cross section analysis presented here measures $\sigma_{\mathrm{pp} \rightarrow \mathrm{tt}}$ with respect to the kinematic properties of the top quark in the dilepton channel, including the effects of higher-order perturbative QCD and electroweak corrections as shown in the right panel of Fig. 2 [6]. Updated results are available in [7]. By applying the corrections the observed trend flattens, however it is not covered completely.
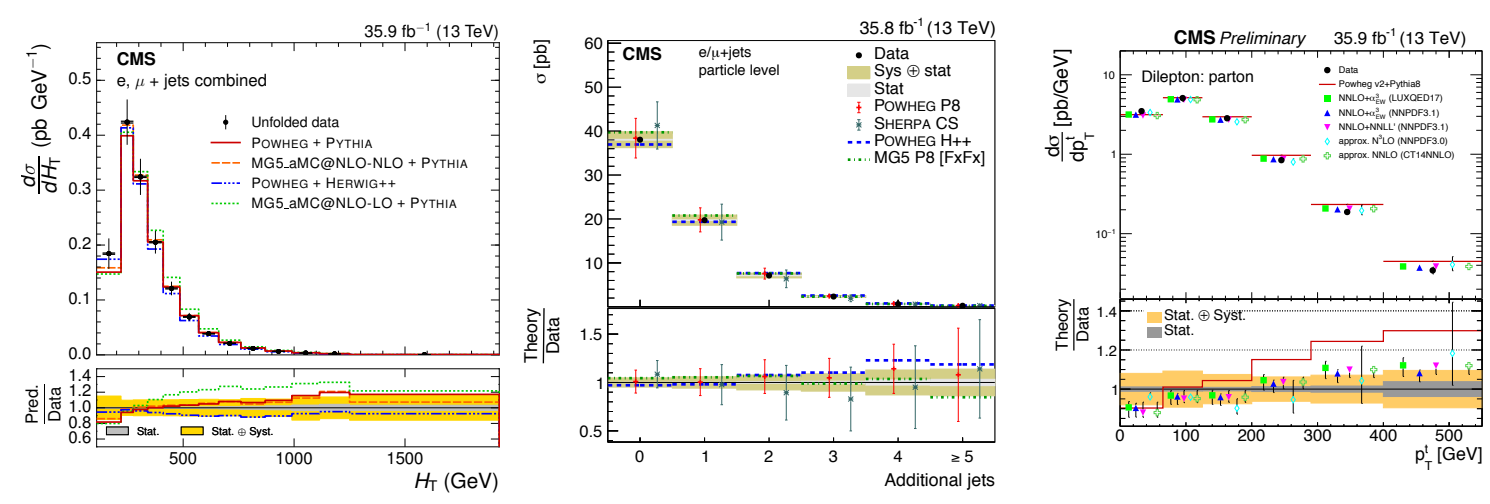

Figure 2: A set of absolute differential cross section measurements with respect to the $H_{\mathrm{T}}$ [4], additional jet multiplicity [5] and $p_{\mathrm{T}}^{t}[6]$ variables. The right plot corresponds to the dilepton final state and is presented at parton level, while the other two correspond to the single lepton final state at particle level.

\section{References}

[1] CMS Collaboration, The CMS Experiment at the CERN LHC, JINST 3 (2008) S08004.

[2] CMS Collaboration, Measurement of the inclusive $\mathrm{t} \overline{\mathrm{t}}$ cross section in pp collisions at $\sqrt{\mathrm{s}}=5.02 \mathrm{TeV}$ using final states with at least one charged lepton, JHEP 03 (2018) 115 [1711.03143].

[3] CMS Collaboration, Observation of top quark production in proton-nucleus collisions, Phys. Rev. Lett. 119 (2017) 242001 [1709.07411].

[4] CMS Collaboration, Measurements of differential cross sections of top quark pair production as a function of kinematic event variables in proton-proton collisions at $\sqrt{s}=13 \mathrm{TeV}, \mathrm{JHEP} 06$ (2018) 002 [1803.03991].

[5] CMS Collaboration, Measurement of differential cross sections for the production of top quark pairs and of additional jets in lepton+jets events from pp collisions at $\sqrt{s}=13$ TeV, Phys. Rev. D 97 (2018) 112003 [1803.08856].

[6] CMS Collaboration, Measurements of differential cross sections for $\overline{\mathrm{t}} \mathrm{p}$ production in proton-proton collisions at $\sqrt{s}=13 \mathrm{TeV}$ using events containing two leptons, CMS-PAS-TOP-17-014, 2018, https://cds.cern.ch/record/2621975.

[7] CMS Collaboration, Measurements of $\mathrm{t} \overline{\mathrm{t}}$ differential cross sections in proton-proton collisions at $\sqrt{s}=$ $13 \mathrm{TeV}$ using events containing two leptons, 1811.06625 , Submitted to JHEP. 